\title{
TESTE DE ZULLIGER NA AVALIAÇÃO DA PERSONALIDADE: UMA PERSPECTIVA HISTÓRICA E SUA APLICABILIDADE NO PROCESSO AVALIATIVO PARA PORTE DE ARMA DE FOGO
}

\section{ZULLIGER TEST IN PERSONALITY ASSESSMENT: A HISTORICAL PERSPECTIVE AND ITS APPLICABILITY IN THE EVALUATION PROCESS FOR CARRYING A FIREARM}

\section{TEST DE ZULLIGER EN LA EVALUACIÓN DE LA PERSONALIDAD: UNA PERSPECTIVA HISTÓRICA Y SU APLICABILIDAD EN EL PROCESO DE EVALUACIÓN DEL PORTE DE ARMA DE FUEGO}

\author{
Astrid Sharon Pontes Hasbun¹, Ionara Dantas Estevam², Nilton Soares Formiga ${ }^{3}$
}

\author{
Submetido em: 04/04/2021 \\ Aprovado em: 24/04/2021
}

\begin{abstract}
RESUMO
A avaliação psicológica é uma atividade ampla e complexa que envolve tanto a pesquisa e construção de instrumentos e técnicas psicológicas quanto o processo de coleta de dados com o intuito de obter informações acerca de um indivíduo, um grupo ou situações específicas, e auxiliando em processos de tomada de decisões em diversos cenários da práxis do profissional psicólogo. Dentre os instrumentos utilizados nos processos de avaliação psicológica está o Teste de Zulliger ( $Z$ teste) que surge da necessidade de realizar avaliações breves de personalidade em contextos que estavam envolvidos diversos indivíduos, revelando-se um teste rico e de fácil utilização, podendo ser usado em diferentes contextos. Sendo assim, em cenários como avaliação para porte de arma de fogo o $Z$ teste é um dos instrumentos mais utilizados em função da rapidez, economia e descrição das características de personalidade dos candidatos. Atualmente a instrução normativa (IN) do departamento da Polícia Federal (DPF) no 78/2014 traz indicadores que devem ser avaliados e que estão divididos em duas categorias: necessários e restritivos. $O Z$ teste tem em suas análises vários dos indicadores elencados na IN DPF no 78/2014 como adaptação, autocontrole, controle emocional, adaptação social, empatia, relacionamento interpessoal, depressão, bem como os relacionados aos transtornos mentais. Entretanto, mesmo havendo esse perfil "pré-definido" e essa seja a referência utilizada hoje para as tomadas de decisão por psicólogos em todo Brasil, esse perfil traçado necessita de maior embasamento científico.
\end{abstract}

\footnotetext{
1 Mestranda em Psicologia pela Universidade Potiguar. Pós-graduada em Avaliação Psicológica pelo Centro Universitário do Rio Grande do Norte. Pós-graduada em Gestão de Pessoas pelo Centro Universitário do Rio Grande do Norte. Graduada em Psicologia pela Universidade Federal do Rio Grande do Norte. Psicóloga credenciada pela Polícia Federal em Avaliação Psicológica para aquisição de porte de arma. Carreira desenvolvida na área de Gestão de Pessoas e educação. Experiência em Avaliação Psicológica para diversos fins como seleção, porte de arma e orientação vocacional. Atualmente é Coordenadora Acadêmica do curso de Psicologia da Universidade Potiguar - Laureate Universities.

2 Psicóloga, possui Doutorado em Psicologia Social pela Universidade Federal da Paraíba, Mestrado em Psicologia pela Universidade Federal da Paraíba, Especialização em Desenvolvimento Infantil e seus Desvios pela Universidade Federal da Paraíba, Especialista em Psicologia Clínica e Psicologia do Trânsito pelo Conselho Federal de Psicologia, Graduação em Psicologia pelo Centro Universitário de João Pessoa- UNIPÊ, Graduação em Licenciatura em Psicologia pelo Centro Universitário de João Pessoa-UNIPÊ. Professora e Pesquisadora da Pós-Graduação Stricto Sensu, Lato Sensu e Graduação de Psicologia da Universidade Potiguar - RN. Professora da Faculdade Mauricio de Nassau - RN. Diretora Técnica da Symmetric - Consultoria em Avaliação Psicológica. Experiência na área de Avaliação Psicológica, Psicologia Clínica e Jurídica. Atuando principalmente nas temáticas de Avaliação Psicológica (Clínica, Pericial, Orientação Profissional e do Trabalho) e Saúde do Trabalhador

${ }^{3}$ Mestrado em Psicologia (Psicologia Social) pela UNIVERSIDADE FEDERAL DA PARAÍBA. Em 2010 doutorouse, também, na UNIVERSIDADE FEDERAL DA PARAÍBA, na pós-graduação em Psicologia Social. Atualmente, é professor/pesquisador no programa de pós-graduação (mestrado) em psicologia organizacional e do trabalho na Universidade Potiguar (UNP) /Laureate International Universities.
} 
PALAVRAS-CHAVE: Avaliação psicológica. Teste de Zulliger. Z teste. Avaliação psicológica para porte de arma de fogo.

\begin{abstract}
Psychological assessment is a broad and complex activity that involves both the research and construction of psychological instruments and techniques, and the process of data collection in order to obtain information about an individual, a group, or specific situations, and assisting in decision making processes in various psychological assessment scenarios. Among the instruments used in psychological assessment processes is the Zulliger Test ( $Z$ test), which arose from the need to perform brief personality assessments in contexts where several individuals were involved, proving to be a rich and easy-to-use test that can be used in different contexts. Thus, in scenarios such as the evaluation for carrying a firearm, the $Z$ test is one of the most used instruments because of its speed, economy, and description of the personality characteristics of the candidates. Currently the Normative Instruction (IN) of the Federal Police Department (DPF) No. 78/2014 brings indicators that must be evaluated and are divided into two categories: necessary and restrictive. The $Z$ test has in its analysis several of the indicators listed in IN DPF no. 78/2014 such as adaptation, self-control, emotional control, social adaptation, empathy, interpersonal relationships, depression, as well as those related to mental disorders. However, even though there is this "pre-defined" profile and it is the reference used today for decision-making by psychologists throughout Brazil, this outlined profile needs more scientific basis.
\end{abstract}

KEYWORDS: Psychological evaluation. Zulliger test. Z test. Psychological evaluation for carrying a firearm.

\title{
RESUMEN
}

La evaluación psicológica es una actividad amplia y compleja que implica tanto la investigación y construcción de instrumentos y técnicas psicológicas como el proceso de recogida de datos con el fin de obtener información sobre un individuo, un grupo o situaciones concretas, y ayudar en los procesos de toma de decisiones en diversos escenarios de la praxis del profesional de la psicología. Entre los instrumentos utilizados en los procesos de evaluación psicológica se encuentra el Test de Zulliger (test $Z$ ) que surge de la necesidad de realizar evaluaciones breves de la personalidad en contextos en los que intervenían varios individuos, resultando ser un test rico y fácil de utilizar que puede ser empleado en diferentes contextos. Así, en escenarios como la evaluación para portar un arma de fuego, el test $Z$ es uno de los instrumentos más utilizados por su rapidez, economía y descripción de las características de personalidad de los candidatos. Actualmente, la instrucción normativa (IN) del Departamento de Policía Federal (DPF) no 78/2014 trae indicadores que deben ser evaluados y se dividen en dos categorías: necesarios y restrictivos. El test $Z$ tiene en su análisis varios indicadores recogidos en el IN DPF no 78/2014 como son la adaptación, el autocontrol, el control emocional, la adaptación social, la empatía, las relaciones interpersonales, la depresión, así como los relacionados con los trastornos mentales. Sin embargo, a pesar de que existe este perfil "predefinido" y que es la referencia utilizada hoy en día para la toma de decisiones por parte de los psicólogos de todo Brasil, este perfil delineado necesita más base científica.

PALABRAS CLAVE: Evaluación psicológica. Test Zulliger. Test Z. Evaluación psicológica para el porte de un arma de fuego.

\section{INTRODUÇÃO}




\section{RECIMA21 - REVISTA CIENTÍFICA MULTIDISCIPLINAR}

Astrid Sharon Pontes Hasbun, Ionara Dantas Estevam, Nilton Soares Formiga

A avaliação psicológica é uma atividade ampla e complexa que envolve tanto a pesquisa e construção de instrumentos e técnicas psicológicas quanto o processo de coleta de dados com o intuito de obter informações acerca de um indivíduo, um grupo ou situações específicas, e auxiliando em processos de tomada de decisões em diversos cenários da práxis do profissional psicólogo (CFP, 2013).

Dentre os instrumentos utilizados nos processos de avaliação psicológica, pode-se mencionar os métodos projetivos, os quais, na concepção de Cunha (1993), especificamente na edição dessa publicação, permite analisar os aspectos estruturais e dinâmicos, mesmo que cada uma delas possa exibir especificidades distintas. Sendo assim, apesar da diversidade do material e formas de interpretação, essas técnicas utilizam a projeção para revelar material dinâmico da psique, seja ele normal ou patológico.

Villemor- Amaral e Resende (2018, p. 127) afirmam que "os testes projetivos avaliam o modo de pensar, sentir e agir do cliente em situações mais complexas, pouco familiares, menos estruturadas e interpessoalmente mais estressantes". São informações que não são facilmente relatadas através da linguagem pois podem estar associadas a afetos ambíguos, e que são mais facilmente acessadas através de imagens relacionadas às situações traumáticas ou não superadas.

Entre as técnicas projetivas está o Teste de Zulliger ( $Z$ teste) criado por Hans Zulliger (1893 1965), psicólogo clínico suíço e amigo de Hermann Rorschach que participou dos seus trabalhos nos experimentos com manchas de tinta feitas ao acaso na avaliação da personalidade.

$\mathrm{O}$ primeiro trabalho baseado em manchas de tinta feito por Zulliger após a morte de Rorschach - Der Rorschasche Testversuch im Dienst der Erziehungsberatung - ocorreu em 1932 e investigava a criatividade e a integração humana. Em 1938 em coautoria com Hans BehnEschenburg publicou BERO (Behn-Rorschach test), constituído igualmente de 10 lâminas contendo manchas feitas ao acaso e construído para ser administrado em crianças. Alguns autores chegaram a afirmar que o BERO seria uma técnica paralela à de Rorschach (Vaz, 2007; Vaz \& Alchieri, 2016).

Considerando a reflexão dos autores supracitados, na época da segunda guerra, Hans Zulliger utilizou a técnica do Rorschach para selecionar oficiais das forças armadas suíças, mas o fator tempo mostrou-se um complicador para as avaliações uma vez que a aplicação com o uso de 10 lâminas despendia muito tempo face ao grande número de pessoas a serem avaliadas.

Hans Zulliger iniciou, então, administrações com novas manchas de tinta em diversos grupos com reduções para 04 lâminas, mas de forma a não afetar a profundidade da investigação. $O$ final das experiências resultou numa amostra de 800 indivíduos e as três lâminas que compõem o $Z$ teste. Daí surge o conjunto para a administração coletiva - Zulliger Diapositive test (1948) e a versão para administração individual com 3 cartões - Der Zulliger-Tafeln-Test (1954) (ver Grazziotin \& Scortegagna, 2016).

Dessa forma, o $Z$ teste surge da necessidade de realizar avaliações breves de personalidade em contextos que estavam envolvidos diversos indivíduos. Zulliger criou, por conseguinte, um 


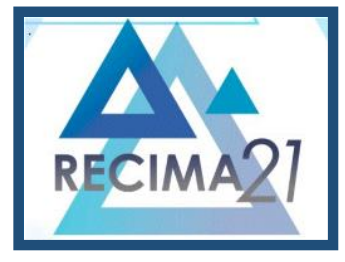

\section{RECIMA21 - REVISTA CIENTÍFICA MULTIDISCIPLINAR}

TESTE DE ZULLIGER NA AVALIAÇÃO DA PERSONALIDADE: UMA PERSPECTIVA HISTÓRICA E SUA APLICABILIDADE NO PROCESSO AVALIATIVO PARA PORTE DE ARMA DE FOGO Astrid Sharon Pontes Hasbun, Ionara Dantas Estevam, Nilton Soares Formiga

instrumento que visava ter as características fundamentais do Rorschach, mas que possibilitava avaliar um grande contingente de pessoas, em menor tempo, de modo rápido e eficaz (Vaz, 2002); com isso, o $Z$ teste revelou-se, então, um teste rico de informação e de fácil utilização, podendo ser usado em diferentes contextos.

O $Z$ teste é, portanto, um instrumento que avalia a personalidade e foi criado com base metodológica no Psicodiagnóstico de Rorschach. Mas qual seria o nome mais adequado? Teste ou técnica de Zulliger? A literatura sobre o tema em questão permite constatar diferentes denominações: a) $Z$ teste; b) Teste $Z$; c) Teste de Zulliger e d) Técnica de Zulliger. A denominação de técnica parece mais adequada pois teste se propõe a medir o que um sujeito é capaz de render ou desempenhar relativizando a um padrão previamente estabelecido e com obtenção de resultado específico. (Vaz, 2007)

As manchas apresentadas nos 03 (três) cartões do $Z$ teste causam nos avaliandos, segundo Vaz e Alchieri (2016) associações percepto-associativas que de alguma forma reproduzem situações internas, seu modo de pensar, de sentir e de tomar decisões. Segundo Villemor-Amaral e Machado (2011), o $Z$ teste examina a estrutura e a dinâmica da personalidade a partir da percepção dos estímulos não estruturados (manchas de tinta) que se apresentam ao sujeito. Essas informações traduzidas nas diversas categorias, determinantes e localizações são quantificadas e interpretadas a partir do dinamismo da personalidade e de sua natureza multifacetada.

As categorias de respostas, localizações e determinantes encontrados no $Z$ teste quando quantificados possibilitam estudos comparativos e correlacionais com possiblidades de predição, entretanto essas categorias devem ser analisadas e avaliadas de forma articulada com outros dados coletados no $Z$ teste e com as dimensões familiar e sociocultural do indivíduo (Vaz \& Alchieri, 2016).

Algumas respostas, por exemplo, podem ser dadas em função da atividade laborativa do indivíduo e isso deve ser levado em consideração na hora da análise das respostas. Dessa forma, embora seja denominado de teste, segundo Vaz e Alchieri (2016) trata-se de uma técnica relativamente não estruturada, multidimensional na mensuração e avaliação psicológica que mobiliza o processo perceptivo-associativo, a imaginação e outros elementos dinâmicos do psiquismo individual.

Para Vaz (2007) é um conjunto de variáveis através das quais se pode quantificar aspectos funcionais e dinâmicos da personalidade. É um método de sentido amplo e não uma unidade de estímulo para obtenção de respostas padrão. Usa-se por tradição o nome $Z$ teste, mas entende-se que se trata de uma técnica relativamente não estruturada.

O $Z$ teste é composto por três diapositivos ou pranchas de 18,5 por $25 \mathrm{~cm}$ com manchas de tinta ambíguas, bilaterais e desestruturadas, sendo uma acromática, uma policromática e outra em preto e vermelho. Ao ser exposto às manchas, a tarefa do sujeito é responder "o que isto poderia ser", de modo livre (Hasbun, Formiga \& Estevam, 2021. p.04) 


\section{RECIMA21 - REVISTA CIENTÍFICA MULTIDISCIPLINAR}

Após a fase de associação, o sujeito inicia a fase de inquérito, respondendo onde está aquilo que viu na mancha e o que tem na mancha que the fez parecer conforme descrito. Em seguida, o teste é codificado, cada elemento da resposta é classificado por uma série de códigos que determinam e qualificam as respostas, como códigos de forma, cor e movimento, por exemplo. Por fim, a frequência de todos os códigos é computada e interpretada, possibilitando fazer a análise de acordo com as tabelas normativas correspondentes. (Hasbun, Formiga \& Estevam, 2021. p.04)

O primeiro diapositivo é uma mancha mais compacta e em função das suas cores acromáticas (cinza, preto e branco) pode causar sensação de angústia. Por ser o primeiro estímulo verifica-se como a capacidade de adaptação do sujeito frente a situações novas e inesperadas. $O$ segundo diapositivo tem cores cromáticas (verde, marrom e tonalidades de vermelho) e possibilita verificar como o sujeito lida com a agressividade, afeto, emoções e impulsividade. O terceiro e último diapositivo traz manchas nas cores preto, vermelho e cinza e traz estímulos para a observação de como o sujeito lida com as relações interpessoais, potencial criativo e empático do sujeito.

A diferença entre o $Z$ teste e o Rorschach é basicamente o número de pranchas, uma vez que a classificação de respostas e análise dos dados se mantém igual ao Rorschach, e a tarefa envolve também a apresentação de estímulos não estruturados que devem ser interpretados, tornando possível obter uma avaliação complexa dos aspectos cognitivos e afetivos a personalidade (Villermor-Amaral \& Machado, 2011).

No Brasil, atualmente, há três versões do $Z$ teste que possuem o parecer favorável pelo Conselho Federal de Psicologia, o Z-Teste coletivo e individual, validado pelos autores Cícero Vaz e João Carlos Alchieri que utiliza o sistema Klopfer para a interpretação dos resultados; o Teste de Zulliger no Sistema Compreensivo - ZSC - forma individual, dos autores Anna Elisa Villemor-Amaral e Ricardo Primi, que se fundamenta no Sistema Compreensivo desenvolvido por Exner para a aplicação, classificação e interpretação dos dados e o teste de Zulliger no sistema Escola de Paris: forma individual dos autores Elizabeth do Nascimento e Marcelo Augusto Resende que se ampara no sistema de correção e interpretação, conhecido inicialmente como "Escola Francesa", idealizado por Ombredane e Canivet. . (Hasbun, Formiga \& Estevam, 2021. p.04)

De acordo com Grazziotin e Scortegagna (2016) a maior parte das pesquisas no Brasil foi realizada no sistema Klopfer até 2004 e a partir de 2005, o ZSC começou a ganhar popularidade. 0 sistema ZSC foi criado na década de 70 por John Exner Jr., com o objetivo de obter uniformidade na aplicação, na codificação e na interpretação do Rorschach.

Para Vaz e Alchieri (2016) as diferenças entre os sistemas são irrelevantes no que se refere ao resultado da avaliação. As diferenças de codificação não implicam discrepâncias na interpretação e diagnóstico. As categorias gerais básicas do Rorschach permanecem.

A técnica utilizada neste estudo é o $Z$ teste sob a perspectiva do Sistema Klopfer, desenvolvido por Bruno Klopfer em 1936 que é um dos modelos norte-americanos que possibilita integração de dados quantitativos e qualitativos. Vaz e Alchieri (2016) sinalizam que Klopfer 


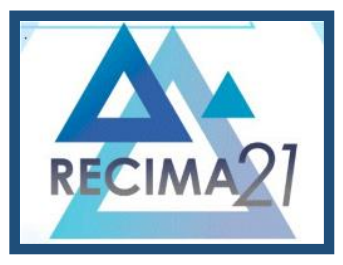

\section{RECIMA21 - REVISTA CIENTÍFICA MULTIDISCIPLINAR}

TESTE DE ZULLIGER NA AVALIAÇÃO DA PERSONALIDADE: UMA PERSPECTIVA HISTÓRICA E SUA APLICABILIDADE NO PROCESSO AVALIATIVO PARA PORTE DE ARMA DE FOGO introduziu alguns elementos básicos diferenciados dos demais sistemas metodológicos como Sombreado de Textura, Cor Simbólica e Movimento Inanimado.

\section{TIPOS DE APLICAÇÃO E FASES DO Z TESTE}

O Z teste pode ser administrado tanto na forma individual quanto coletiva e alguns cuidados são necessários na preparação do ambiente e material utilizado. Independentemente do tipo de aplicação a ser realizada o local deve ser previamente examinado. A sala não deve ter ruídos externos, ter boa luminosidade e adequada circulação de ar (Vaz, 2002, 2007; Vaz \& Alchieri, 2016).

A aplicação coletiva é indicada em casos de seleção de pessoal e pesquisa. É condição mínima que o examinando saiba escrever, pois neste tipo de aplicação a verbalização, assim como o mapeamento das respostas é feito pelo examinando. O número de examinandos não pode ser superior a 35 e aqueles que apresentem problemas de visão devem se sentar nas primeiras fileiras. A sala não deve ser muito grande e deve ter condições de ser escurecida para melhor visualização da projeção das manchas quando a aplicação for realizada durante o dia.

A distância da primeira fileira para a tela deve ser de $2 \mathrm{~m}$ e da última para a tela de $12 \mathrm{~m}$ no máximo. Neste tipo de aplicação a verbalização, assim como o mapeamento das respostas é feito pelo examinando.

O material necessário consiste em: tela clara que não tenha deformidades e com dimensões de 1,6m x 1,25m; CD com a versão eletrônica das manchas de tinta; projetor multimídia ou equipamento correspondente; flipchart para possíveis ilustrações de preenchimento durante as instruções; canetas esferográficas; folhas de administração e folha de mapeamento de respostas.

A aplicação coletiva se divide em quatro fases:

a) Rapport: o psicólogo deve proporcionar ao grupo um clima de espontaneidade e de estar à vontade. Não há um roteiro padrão, importante que seja uma conversa leve e informal fazendo com que os examinandos se sintam bem para realização a atividade;

b) Instruções: após a fase de rapport e o examinador dará as instruções que constam no manual do $Z$ teste;

c) Aplicação propriamente dita: após perceber que todos entenderam as instruções, o examinador começa a projeção da primeira mancha de tinta seguindo o tempo determinado para a projeção e em seguida para o registro das respostas por parte dos examinandos. Repete o mesmo processo para as manchas 2 e 3 ;

d) Mapeamento do que foi sugerido: O examinador volta a projetar as manchas na tela, uma por uma, solicitando que cada examinando indique onde viu o que registrou na fase de aplicação. Ele deverá contornar a área onde se situa o que imaginou e que está anotado na folha de administração. O processo será repetido para as imagens 2 e 3 . Após essa fase, o psicólogo pode conversar um pouco com os examinandos de como eles se sentiram durante a aplicação tentando diminuir possíveis ansiedades geradas pela aplicação do teste. 


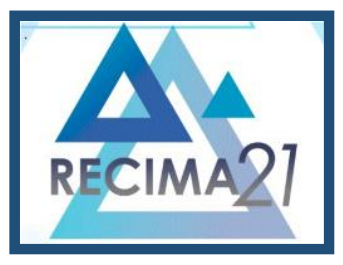

\section{RECIMA21 - REVISTA CIENTÍFICA MULTIDISCIPLINAR}

TESTE DE ZULLIGER NA AVALIAÇÃO DA PERSONALIDADE: UMA PERSPECTIVA HISTÓRICA E SUA APLICABILIDADE NO PROCESSO AVALIATIVO PARA PORTE DE ARMA DE FOGO Astrid Sharon Pontes Hasbun, Ionara Dantas Estevam, Nilton Soares Formiga

Já a aplicação individual propicia uma coleta de dados mais minuciosa sobre a personalidade do examinando. Neste tipo de aplicação a sala pode ser relativamente pequena com espaço para uma mesa para a administração e duas cadeiras. Diferente da aplicação coletiva, neste tipo de aplicação quem faz as anotações é o examinador. O material necessário consiste em: conjunto dos três diapositivos do $Z$ teste; protocolo de mapeamento das respostas; canetas com cores diferentes; folhas de papel pautado e cronômetro (opcional).

A aplicação individual também tem quatro fases:

a) Rapport: mesmas orientações da aplicação coletiva;

b) Instruções: as instruções são diferentes da aplicação coletiva e devem seguir o que é sugerido no manual do $Z$ teste;

c) Aplicação propriamente dita: O examinador entrega o primeiro diapositivo ao examinando e anota todas as verbalizações. Repete o processo para os diapositivos 2 e 3;

d) Fase de inquérito: Após o examinando devolver o $3^{\circ}$ e último diapositivo o examinador inicia o inquérito. $O$ objetivo dessa fase é entender um pouco melhor cada resposta dada pelo examinando para que seja possível identificar bem os conteúdos verbalizados e diferenciar o que influenciou no seu processo perceptivo-associativo. É preciso ficar claro que essa não é uma checagem de respostas certas ou erradas e deve-se ter cuidado para não induzir o examinando. É nessa fase também que o examinador checa a localização de cada resposta dada pelo examinando.

\section{CLASSIFICAÇÃO DAS RESPOSTAS E INTERPRETAÇÃO BÁSICA SEGUNDO O SISTEMA KLOPFER}

\section{Respostas}

A resposta é o conteúdo verbalizado pelo examinando. De acordo com Vaz (2007, p.389) "é uma ideia, um conceito vinculado diretamente a determinada área-estímulo do diapositivo". $\mathrm{O}$ examinando dá à aquela área do diapositivo uma característica, um nome, um conteúdo. Importante salientar que comentários não são considerados como respostas.

Para Vaz e Alchieri (2016) a resposta deve responder a seguinte pergunta: "O que o examinando viu na figura ou em partes da figura?". Ainda segundo os autores, o total de respostas pode ser interpretado como a expressão de capacidade de produção, desempenho e de adaptação à tarefa. Cada resposta é classificada quanto a: 1) localização; 2) Determinantes; 3) Conteúdos e 4) Fenômenos especiais.

\section{Localizações}

As localizações são as áreas onde o examinando situa suas respostas e dependendo da sua localização a resposta será classificada em global $(G)$, detalhe comum (D) e detalhe incomum (Dd). 


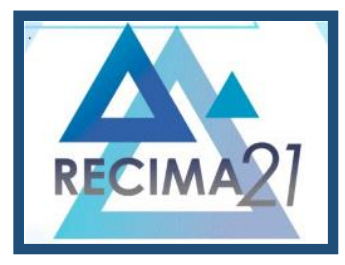

\section{RECIMA21 - REVISTA CIENTÍFICA MULTIDISCIPLINAR}

TESTE DE ZULLIGER NA AVALIAÇÃO DA PERSONALIDADE: UMA PERSPECTIVA HISTÓRICA E SUA APLICABILIDADE NO PROCESSO AVALIATIVO PARA PORTE DE ARMA DE FOGO Astrid Sharon Pontes Hasbun, Ionara Dantas Estevam, Nilton Soares Formiga

Para realizar essas classificações sugere-se fazer o uso do atlas de localizações da técnica de Zulliger que consta no manual do $Z$ teste.

De acordo com Vaz e Alchieri (2016) pode-se dizer que as categorias de localização significam o modo como a pessoa percebe racionalmente a realidade. Elas expressam como a pessoa usa a sua inteligência: a) percepção de síntese e capacidade de planejamento nas repostas denominadas globais; b) capacidade de discriminação perceptiva e senso de objetividade nas respostas em áreas de detalhe comum; c) capacidade de análise e senso de observação nas respostas de detalhe incomum. A presença de respostas com espaço branco combinado com as categorias de localização pode remeter a interferência de ansiedade situacional.

\section{Determinantes}

Os determinantes são os elementos mais complexos da classificação e identificam quais características da mancha estimularam o examinando e influenciaram na formação da resposta. Para Vaz (2007) os determinantes mobilizam o mundo interno do sujeito através das manchas. Eles são a expressão das experiências passadas e projetadas pelo sujeito nas pranchas. Pode-se dizer que é a relação que estabelece entre o mundo externo e o seu mundo interno através das manchas. São eles:

a) Forma (F)

Conteúdo percebido pelo examinando apenas pela forma da mancha. Neste tipo de resposta não há combinação de cor, ação ou sombreado. Pode ser classificada em F+, F+- ou F-.

De acordo com Vaz e Alchieri (2016) o somatório do determinante F é indicativo da expressão da capacidade de perceber as coisas como elas se apresentam na realidade e sem interferência prejudicial das emoções. Um somatório elevado de $\mathrm{F}$ é sinal de alerta para controle demasiado e repressão das emoções. Já um percentual baixo ocorre comumente em casos de pobreza intelectual.

b) Movimento (M, FM, Fm, mF, m)

Respostas de movimento humano são conteúdos percebidos como ação vital podendo ser de deslocamento, de extensão e de postura ou flexão. Pode ser classificado em M+, M+- ou M-.

Para Vaz (2007) o movimento humano tem sido interpretado como criatividade, capacidade de integração e empatia. Um índice elevado de $M$ pode significar inteligência criativa ou ainda reações do tipo maníaco. Já a ausência de $M$ frequentemente ocorre em casos de pessoas ansiosas, tensas, inibidas ou depressivas.

Há também as respostas de movimento animal (FM) como indicativo de impulsividade ou dinamismo. Segundo Vaz e Alchieri (2016) a verbalização de conteúdo animal está relacionada à infância e verifica aspectos relacionados à iniciativa e capacidade de competição.

Respostas de movimento inanimado ( $\mathrm{Fm}, \mathrm{mF}$ ou $\mathrm{m}$ ) são aquelas em que o conteúdo é visto em movimento, mas a ação não é de humano ou animal, mas é movido por forças físicas, químicas, 


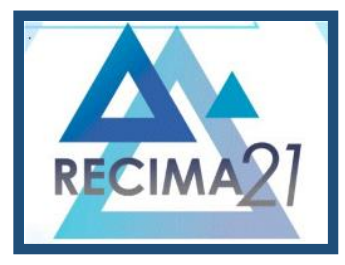

\section{RECIMA21 - REVISTA CIENTÍFICA MULTIDISCIPLINAR}

TESTE DE ZULLIGER NA AVALIAÇÃO DA PERSONALIDADE: UMA PERSPECTIVA HISTÓRICA E SUA APLICABILIDADE NO PROCESSO AVALIATIVO PARA PORTE DE ARMA DE FOGO mecânicas ou abstratas. Vaz (2007) sinaliza que o somatório de movimento inanimado tem significado de conflitos internos com os quais a pessoa está com dificuldades para lidar.

c) Cor cromática (FC, CF, C, Cn, F-C, C-F, F/C, C/F, Csimb)

De acordo com Vaz (2007) as cores são um dos aspectos mais relevantes no $Z$ teste porque mobilizam o mundo interno da pessoa provocando reações emocionais seja de aproximação ou de afastamento. Considera-se respostas de cores cromáticas aquelas em que o examinando formula a resposta levado pelo colorido da mancha. São classificadas dependendo da precisão da resposta e da ênfase dada à cor ou à forma e da compatibilidade ou não da resposta com a cor. Representam capacidade de receber e retribui afeto e reação a estímulos emocionais.

d) Cor acromática (C', C'F, FC',)

São respostas em que aparecem o preto e o cinza e são interpretadas como tendência a evitar estímulos que possam mobilizar reações emocionais. Para Vaz (2007) as cores acromáticas expressam depressão como traço de personalidade e não como reação transitória. As classificações em relação à forma seguem os mesmos princípios das cores cromáticas.

e) Sombreado Radiológico e Perspectiva (Fk, kF, k, FK, KF, K)

O sombreado de perspectiva são respostas que envolvem percepção tridimensional, que dão ideia de perspectiva, de profundidade. Segundo Vaz e Alchieri (2016) expressam a capacidade de adaptação afetiva, cautelosa para melhor suportar o sofrimento e evitar a depressão.

Já o sombreado radiológico está presente em conteúdos vistos em radiografias, transparências e fotografias. É a extensão tridimensional projetada em um plano bidimensional.

São dois grupos de determinantes que de acordo com Vaz e Alchieri (2016) não aparecem com frequência na aplicação coletiva, sendo mais comum na individual. Recomenda-se, entretanto verificar como está o controle e as condições afetivas nas outras variáveis para entender melhor como o indivíduo procura meios de adaptação ou ajustamento quando enfrenta uma situação ansiogênica.

f) Textura (Fc, cF, c)

Algumas pessoas ao dar a resposta friccionam a mancha como se ali houvesse alguma textura. No $Z$ teste coletivo é difícil de perceber esse determinante seja pela projeção ou por não haver o momento de inquérito por parte do examinador. A resposta de textura é uma forma de expressão das condições afetivas que busca afeto em alguém ou alguma coisa. As classificações em relação à forma seguem os mesmos princípios das cores cromáticas.

\section{Conteúdos}

Refere-se ao que foi verbalizado, percebido, o que o sujeito viu. Os principais conteúdos são: Conteúdo humano $(\mathrm{H}, \mathrm{Hd})$; conteúdo humano descaracterizado $((\mathrm{H}),(\mathrm{Hd}))$; conteúdo animal $(\mathrm{A}, \mathrm{Ad})$ e respostas com conteúdo anatômico (Anat). Cabe uma ressalva para os casos de examinandos que 


\section{RECIMA21 - REVISTA CIENTÍFICA MULTIDISCIPLINAR}

TESTE DE ZULLIGER NA AVALIAÇÃO DA PERSONALIDADE: UMA PERSPECTIVA HISTÓRICA E SUA APLICABILIDADE NO PROCESSO AVALIATIVO PARA PORTE DE ARMA DE FOGO Astrid Sharon Pontes Hasbun, Ionara Dantas Estevam, Nilton Soares Formiga

trabalham na área da saúde que pela prática tendem a dar mais respostas de conteúdo anatômico (Vaz \& Alchieri, 2016)

\section{Fenômenos Especiais}

Os fenômenos especiais são, de acordo com Vaz e Alchieri (2016), manifestações não quantificáveis através das verbalizações do examinando, mas que devem ser levados em consideração para o entendimento do funcionamento do mundo interno do examinando. Dentre os fenômenos especiais destacam-se (ver quadro 1):

Quadro 1: Principais Fenômenos Especiais

\begin{tabular}{|c|c|}
\hline Fenômeno Especial & Características \\
\hline Choque ao branco & $\begin{array}{l}\text { Preocupação do examinando com o branco ao redor } \\
\text { (exterior) das manchas }\end{array}$ \\
\hline Choque ao vazio & $\begin{array}{l}\text { Preocupação do examinando com os espaços em branco do } \\
\text { interior das manchas. }\end{array}$ \\
\hline Choque acromático & $\begin{array}{l}\text { Dificuldades do examinando em relação às manchas escuras } \\
\text { do diapositivo I e III. }\end{array}$ \\
\hline Choque cromático & $\begin{array}{l}\text { Perturbação sentida e manifestada pelo examinando diante } \\
\text { dos estímulos coloridos dos diapositivos II e III. }\end{array}$ \\
\hline Choque que exclamação & São expressões de emoção como: Hum, Ih. Bah. \\
\hline Confabulação & $\begin{array}{l}\text { Caracterizada por fantasia e divagação verbal com uso de } \\
\text { imaginação fantasiosa em torno do que está sendo falado. }\end{array}$ \\
\hline Contaminação & $\begin{array}{l}\text { Examinando inicia a verbalização a partir de um detalhe da } \\
\text { mancha, tenta integrar detalhes que vai percebendo, só que } \\
\text { de forma incoerente e ilógica. }\end{array}$ \\
\hline Crítica à técnica & Expressão de crítica às manchas ou ao teste como um todo. \\
\hline Ideia de autorreferência & $\begin{array}{l}\text { Examinando identifica coisas suas, de sua propriedade, } \\
\text { partes de si na mancha. }\end{array}$ \\
\hline Ideia de referência & $\begin{array}{l}\text { Preocupação do examinando em descrever as verbalizações } \\
\text { como coisas identificadas com as de seu relacionamento. }\end{array}$ \\
\hline Linguajar requintado & Expressões rebuscadas, formalismos e "estrangeirismos". \\
\hline Mutilação & $\begin{array}{l}\text { Expressão de membros de animais ou pessoas quebradas, } \\
\text { acidentados, machucados e cortados. }\end{array}$ \\
\hline Sentimento de incapacidade & $\begin{array}{l}\text { Pode ser manifestado através de expressões como "não dá", } \\
\text { "tá difícil", "não posso", "não consigo". }\end{array}$ \\
\hline
\end{tabular}

Nota: Elaborado pela autora. Fonte: Vaz e Alchieri (2016). 


\section{RECIMA21 - REVISTA CIENTÍFICA MULTIDISCIPLINAR}

TESTE DE ZULLIGER NA AVALIAÇÃO DA PERSONALIDADE: UMA PERSPECTIVA HISTÓRICA E SUA APLICABILIDADE NO PROCESSO AVALIATIVO PARA PORTE DE ARMA DE FOGO Astrid Sharon Pontes Hasbun, Ionara Dantas Estevam, Nilton Soares Formiga

\section{ZTESTE PARA PORTE DE ARMA DE FOGO}

De acordo com Resende, Rodrigues e Silva (2008) o uso de arma de fogo exige, de quem a porta, características de personalidade como controle das emoções, da impulsividade e da agressividade. Além disso, é necessária adequação à realidade e adaptação social, já que o uso indevido pode provocar a morte de si mesmo e a de terceiros.

Para Resende (2012) o $Z$ teste é um dos instrumentos mais utilizados em função da rapidez, economia e descrição das características de personalidade dos candidatos ao manuseio de arma de fogo. Além de fornecer uma análise detalhada, possibilitando uma avaliação da vida emocional, dos controles, da adaptação social, da adequação à realidade, dos conflitos, aspirações, potencial intelectual, nível de angústia, entre outros.

Segundo o autor supracitado, dentre os aspectos de personalidade sugere-se importante analisar os aspectos de agressividade, capacidade de adaptação, impulsividade, ajustamento pessoal e social, expressão de afetos, nível de maturidade e autopercepção.

Além disso, é importante avaliar a posição às normas sociais e figuras de autoridade, relacionamentos interpessoais, exteriorização de reações afetivas, expressão de raiva, vulnerabilidade, nível de angústia, conflitos, ansiedade, depressão e transtornos psíquicos.

Segundo Resende (2016) a polícia federal estabeleceu em 1998 alguns critérios específicos, valores médios no $Z$ teste, e que deveriam ser considerados na tomada de decisão das avaliações psicológicas para manuseio de arma de fogo. São eles: número de respostas ( 15 a 22 na aplicação individual e 8 a 12 na aplicação coletiva), globais (16 a 20\%), detalhes comuns (60 a 68\%), detalhes incomuns (4 a 13\%), respostas no branco (12\%), controle intelectual (45 a 55\%), senso crítico (65 a $80 \%$ ), recursos internos (80 a $90 \%$ ), controle externo das reações afetivas (2:1), controle interno (2:1), controle social (2:1), controle geral (65 a $80 \%$ ), nível de aspiração (2:1), flexibilidade do pensamento (35 a 55\%), capacidade de relacionamento (20 a $30 \%$ ), participação no pensamento do grupo (20 a 30\%) e controle da ansiedade (12\%). De acordo com o autor não foi descrita a fonte destas informações ou o sistema de correção utilizado.

Atualmente a instrução normativa (IN) do departamento da Polícia Federal (DPF) ㄲo 78/2014 traz indicadores que devem ser avaliados e que estão divididos em duas categorias: necessários (adaptação, atenção, autocontrole, afetividade, autocrítica, concentração, controle emocional, decisão, empatia, energia, equilíbrio, estabilidade, flexibilidade, maturidade, memória, meticulosidade, percepção, prudência, relacionamento interpessoal, resistência à frustração, segurança, senso crítico, sociabilidade) e restritivos (Reações relacionadas aos transtornos: mentais causados por uma condição médica geral; relacionados a substâncias; somatoformes; factícios; dissociativos; do humor; de ansiedade; da personalidade; preconceito, fanatismo) (Brasil, 2014).

$\mathrm{O} Z$ teste tem em suas análises vários desses indicadores de aptidão e inaptidão que estão listados na IN DPF no 78/2014 como adaptação, autocontrole, controle emocional, adaptação social, 


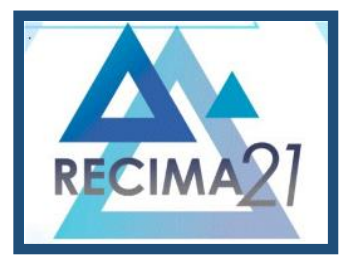

\section{RECIMA21 - REVISTA CIENTÍFICA MULTIDISCIPLINAR}

TESTE DE ZULLIGER NA AVALIAÇÃO DA PERSONALIDADE: UMA PERSPECTIVA HISTÓRICA E SUA APLICABILIDADE NO PROCESSO AVALIATIVO PARA PORTE DE ARMA DE FOGO Astrid Sharon Pontes Hasbun, Ionara Dantas Estevam, Nilton Soares Formiga

empatia, relacionamento interpessoal, depressão, bem como os relacionados aos transtornos mentais. A compreensão do significado de cada desses indicadores é imprescindível para que o psicólogo possa fazer uma avaliação psicológica para concessão do porte de arma de fogo com a utilização do $Z$ teste.

Mesmo havendo esse perfil "pré-definido" e essa seja a referência utilizada hoje para as tomadas de decisão por psicólogos em todo Brasil, esse perfil traçado necessita de maior embasamento científico. Reforçando essa percepção, Maya (2017) afirma que na instrução normativa consta apenas indicadores de personalidade necessários e restritivos, porém sem especificar quanto a presença de um ou de outro traço é suficiente para a indicação ou contraindicação.

Observa-se, portanto, que nos estudos analisados busca-se construir um perfil favorável para o portador da arma de fogo. Mas o grande desafio é tentar traçar também o perfil restritivo, e quanto cada indicador desse pode estar presente em um protocolo favorável ao porte de arma, pois a partir dos estudos atuais não há possibilidade de afirmar quais construtos de fato tornam o indivíduo inapto para o manuseio de arma de fogo.

\section{CONSIDERAÇÕES FINAIS}

O Z teste é um dos instrumentos mais utilizados nas avaliações psicológicas para porte de arma de fogo em função da rapidez, economia na aplicação e descrição das características de personalidade dos candidatos ao manuseio de arma de fogo.

Entretanto, é importante ressaltar que em um processo de avaliação psicológica, um teste não é valido por si só, por mais robusta que seja a validade teórica do instrumento, deve considerar sua validade relacionada ao contexto em que se pretende utilizar e da fidedignidade dos seus resultados.

De forma específica para avaliações no contexto de porte de arma de fogo se faz necessário estudos que possibilitem a elaboração de um perfil do candidato, ou ainda um protocolo de padronização que possa dar maior segurança ao profissional que atua na área dada a responsabilidade e repercussão social desta atuação.

Desta forma sugere-se que se dê continuidade com pesquisas utilizando o teste Zulliger no contexto da avaliação para porte de arma, almejando demonstrar evidências de validade mais robustas, assim como se faz necessário expandir os estudos para diferentes regiões geográficas do Brasil para que possamos ter uma adequação do instrumento para as diversas realidades encontradas no país.

\section{REFERÊNCIAS}




\section{RECIMA21 - REVISTA CIENTÍFICA MULTIDISCIPLINAR}

TESTE DE ZULLIGER NA AVALIAÇÃO DA PERSONALIDADE: UMA PERSPECTIVA HISTÓRICA E SUA APLICABILIDADE NO PROCESSO AVALIATIVO PARA PORTE DE ARMA DE FOGO Astrid Sharon Pontes Hasbun, Ionara Dantas Estevam, Nilton Soares Formiga

BRASIL. Polícia Federal. Instrução normativa no.78/2014-DG/DPF de 10 de fevereiro de 2014. Estabelece procedimentos para 0 credenciamento e fiscalização de psicólogos responsáveis pela expedição de comprovante de aptidão psicológica para o manuseio de arma de fogo e regulamenta a atuação do psicólogo na avaliação psicológica do vigilante, 2014. Disponível em: http://www.pf.gov.br/servicos-pf/armas/credenciamento-psicologos/psicologos-crediciados/IN\%20782014.docx/view. Acesso em: 18 abr. 2021.

CONSELHO FEDERAL DE PSICOLOGIA. Cartilha Avaliação Psicológica. Brasília: CFP, 2013 Disponível em: http://satepsi.cfp.org.br/docs/cartilha.pdf Acesso em: 18 abr. 2021.

CUNHA, J. A. Psicodiagnóstico. 4. ed. Porto Alegre: Artes médicas, 1993. Cap. 6. p. 101-133

GRAZZIOTIN, J. B. D.; SCORTEGAGNA, S. A. Mediação no Zulliger: Evidências de Validade em Amostra de Não Pacientes. Psico-USF, v. 21, n. 1, p. 63-72, 2016. DOI: https://doi.org/10.1590/141382712016210106

HASBUN, A. S. P.; FORMIGA, N. S.; ESTEVAM, I. D. Z test and decision making: Ideographic psychological assessment for firearm handling. Research, Society and Development, v. 10, n. 4, p. e5910413815, 2021. DOI: 10.33448/rsd-v10i4.13815. Disponível em: https://rsdjournal.org/index.php/rsd/article/view/13815. Acesso em: 18 abr. 2021.

MAYA, A. S. Avaliação psicológica para manuseio de arma de fogo: considerações críticas à IN-DPF № 78 de 10.02.2014. Especialize, Goiania, v. 14, n. 01, 2017. Disponível em: https://www.ipog.edu.br/revista-especialize-online/edicao-n14-2017/avaliacao-psicologica-paramanuseio-de-arma-de-fogo-consideracoes-criticas-a-in-dpf-n-78-de-10-02-2014/ Acesso em: 18 abr. 2021.

RESENDE, M. A.; RODRIGUES, C. T.; SILVA, C. A. Avaliação da personalidade: técnicas e contextos diversos. In.: V Encontro da Associação Brasileira de Rorschach e Métodos Projetivos, Ribeirão Preto/SP, 2008.

RESENDE, M. A. O teste de Zulliger na avaliação para o porte de arma de fogo. In: VI Congresso da Associação Brasileira de Rorschach e Métodos Projetivos, Brasília/DF, 2012.

RESENDE, M. A. O Teste de Zulliger - estudo psicométrico para concessão de porte de arma de fogo e normatização com adultos de Belo Horizonte (MG). Tese (Doutorado)-Faculdade de Filosofia e Ciências Humanas, Universidade Federal de Minas Gerais, Belo Horizonte, 2016. Disponível em: $\quad$ http://www.fafich.ufmg.br/pospsicologia/wp-content/plugins/downloadattachments/includes/download.php?id=1926. Acesso em: 18 abr. 2021.

VAZ, C. E. Z teste: técnica de Zulliger: forma coletiva. São Paulo: Casa do Psicólogo, 2002.

VAZ, C. E. Psicodiagnóstico. V. 5. ed. Porto Alegre: Artmed, 2007. Cap. 24. p. 386-398.

VAZ, C. E.; ALCHIERI, J. C. Z teste: coletivo e individual: técnica de Zulliger. 3. ed. São Paulo: Hogrefe CETEPP, 2016.

VILLEMOR-AMARAL, A. E.; MACHADO, M. S. Indicadores de depressão do Zulliger no Sistema Compreensivo (ZSC). Paidéia, Ribeirão Preto, v. 21, n. 48, p. 21-27, 2011. DOI: https://dx.doi.org/10.1590/S0103-863X2011000100004.

VILLEMOR-AMARAL, A. E.; RESENDE, A. C. Novo Modelo de Avaliação Psicológica no Brasil. Psicologia: Ciência e Profissão, v. 38, Especial, p. 122-132, 2018. DOI: https://dx.doi.org/10.1590/1982-3703000208680 